Lena-Lisa Wüstendörfer

\title{
Streit um Fidelio. Gustav Mahler und \\ Felix Weingartner im Disput um Werktreue
}

Die Rezeption von Ludwig van Beethovens einziger Oper Fidelio erlebte zu Beginn des 20. Jahrhunderts einen Aufschwung: Maßgebend für das neu geweckte Interesse waren mit Gustav Mahler und Felix Weingartner zwei der profiliertesten Dirigenten der Zeit. Beide räumten Beethoven in ihrem Konzertrepertoire den Platz des unangefochtenen Spitzenreiters ein, ${ }^{\mathrm{I}}$ und für beide hatte Fidelio in ihrer persönlichen Wertschätzung einen besonders hohen Stellenwert. Ihre Vorstellungen davon, wie eine dem Komponisten angemessene Umsetzung dieser Oper zu gestalten sei, unterschieden sich jedoch grundlegend voneinander. Die Sichtweisen auf Fidelio waren so offensichtlich divergierend, dass die Differenzen in Wien, wo beide aufeinanderfolgend den Posten des Hofoperndirektors bekleideten, nachgerade zum Politikum wurden. Worauf die unterschiedlichen Auffassungen einer adäquaten Fidelio-Produktion gründeten, was der Disput über die Positionierung von Mahler und Weingartner im Spannungsfeld von Text- und Werktreue aussagt und inwiefern diese Ereignisse im Jahrzehnt nach der Jahrhundertwende die Fidelio-Rezeption nachhaltig prägten, soll im Folgenden zur Sprache kommen.

Um I900 zählte Fidelio im Gegensatz zu Beethovens sinfonischem Werk zwar zum Repertoire der Opernhäuser, gelangte jedoch vergleichsweise selten zur Aufführung. Dies änderte sich in Wien mit Gustav Mahlers Wirken als Direktor der Hofoper: Für Mahler stellte Fidelio ein Meisterwerk, wenn nicht gar die wertvollste Oper überhaupt dar. ${ }^{2}$ Ihre wahre Größe ließen seinem Urteil zufolge jedoch erst einige wesentliche Anpassungen der Partitur offenbar werden.

Beethovens auf einem Libretto von Joseph Sonnleithner basierende Oper in zwei Akten beginnt in ihrer letzten Fassung von I8I4 ganz in der Art eines Singspiels mit einer alltäglichen Szene, die Marzelline, die Tochter des Kerkermeisters Rocco, beim Bügeln zeigt. Während sie ihrer Arbeit nachgeht, wird sie vom Pförtner Jaquino umworben. In wortreichem Dialog versucht sie, ihm ihr Desinteresse verständlich zu machen, denn sie

1 Vgl. Silvan Moosmüller: Stiltreues und wirkungstreues Interpretieren. Felix Weingartner, Gustav Mahler und die neunte Symphonie Ludwig van Beethovens, in: Im Mass der Moderne. Felix Weingartner - Dirigent, Komponist, Autor, Reisender, hg. von Simon Obert und Matthias Schmidt, Basel 2009, S.327-349, insb. S.34I.

2 Vgl. Henry-Louis de La Grange: Gustav Mahler, Bd.3: Vienna. Triumph and Disillusion (I904-I907), Oxford/New York 2007 ( ${ }^{\mathrm{I}}$ I999), insb. S. I. 
hat sich in Fidelio, den neuen Gehilfen ihres Vaters, verliebt. Als Fidelio von seinen Besorgungen zurückkehrt, die er zur vollen Zufriedenheit Roccos erledigt hat, verspricht ihm dieser seine Tochter zur Frau.

Für Mahler steht die in seinen Augen belanglose Nebenhandlung des prosaischen Opernbeginns in starkem Kontrast zum eigentlichen Drama um den zu Unrecht eingekerkerten Florestan, welches im Wesentlichen jedoch erst mit dem Auftritt des Gouverneurs Pizarro im weiteren Verlauf des ersten Akts in den Fokus rückt. Durch einen solchen >Stilbruch sah sich Mahler veranlasst, den ursprünglich im Hof des Staatsgefängnisses sich abspielenden ersten Akt in Anlehnung an die erste, dreiaktige Version der Oper von I805 szenisch zweizuteilen und die häusliche Szene des Beginns in die heile Welt einer Stube mit Biedermeier-Interieur zu verlegen. Während die so entstandene Gliederung Rückbezug auf Beethovens erste Werkfassung nahm, stammte die Verlegung des Schauplatzes in die gute Stube von Mahler selbst. Die idyllische Heiterkeit dieses Opernbeginns dämpfte Mahler im Hinblick auf die dramatische Fortsetzung durch weitere Maßnahmen: Er kürzte den als zu trivial empfundenen Text Jaquinos, strich Roccos Gold-Arie (»Hat man nicht auch Gold beineben«) und besetzte die Rolle der Marzelline nicht wie traditionellerweise üblich mit einer Soubrette, sondern dachte sie einem lyrischen Sopran zu. ${ }^{3}$ Der Szenenwechsel in den Gefängnisinnenhof erfolgte sodann ohne musikalischen Unterbruch, während das Orchester zur Ankündigung der mit dem fünften Auftritt eintretenden Soldaten und Pizarros den vorgesehenen Marsch erklingen ließ.

Die aufsehenerregendste Änderung durch Mahler stellte jedoch der Einschub der dritten Leonoren-Ouvertüre mit ihrer Spielzeit von rund einer Viertelstunde dar, die Mahler im zweiten Akt zwischen Kerker- und Schlussszene einfügte. Sie folgte somit direkt auf die Befreiung Florestans, den dramatischen Höhepunkt der Oper, während die Bühne vom unterirdischen Verließ in den Schlossplatz verwandelt wurde. Der Grund für diesen Einschub lag wohl hauptsächlich in der für den aufwändigen Umbau des Bühnenbilds benötigten Zeit. Seine Wirkung ging jedoch über die eines Intermezzos hinaus: Bereits im Verständnis Richard Wagners, der Mahler in mancherlei Hinsicht als Vorbild galt, stellte die große Leonoren-Ouvertüre eine kondensierte Version der Oper in musikalischer Form dar, in der die essenzielle dramatische Entwicklung der Oper von den unmenschlichen Tiefen des Kerkers bis zur umjubelten Befreiung rein instrumental nachempfunden werden konnte. ${ }^{4}$ Durch die Platzierung ebendieser Ouvertüre am dramatischen Wendepunkt der Oper bewirkte Mahler - ob beabsichtigt oder nicht - eine apotheotische Steigerung des Geschehens im Wagner'schen Sinne, beschwerte das Sing-

4 Vgl. Carl Friedrich Glasenapp: Wagner-Encpklopädie, Hildesheim I977 (' $\left.{ }^{\mathrm{I}} 89 \mathrm{~g}\right)$, S. 98. 
spiel von einst mit zusätzlichem Pathos und versah es mit deutlichen Zügen eines Musikdramas. 5

Mahlers Eingriffe in Beethovens Fidelio kamen einer eigentlichen Neugestaltung des Werks gleich. Sie beruhten auf seinem Streben nach einer Einheit von Text, Musik und Handlung im Musiktheater, die dieses gleichsam zum `Gesamtkunstwerk nach Wagners Vorbild werden ließ. ${ }^{6}$ Für die Umsetzung der Vision eines Verschmelzens von musikalischem Geschehen und szenischer Darstellung erwiesen sich die Verhältnisse und Arbeitsprozesse, wie Mahler sie I897 bei Amtsantritt an der Wiener Hofoper vorfand, jedoch als ungeeignet: Hier bestimmten die »Vorstände des Kostüm- und Ausstattungswesens [...], durch die Tradition bevollmächtigt, weitgehend selbständig [...], was aus dem Fundus genommen, was neu anzufertigen « war,7 während die Abläufe auf der Bühne »oft lediglich von einem ausgedienten Sänger« geführt wurden, der die »Auf- und Abtritte der Mitwirkenden « regelte. ${ }^{8}$ Um eine Einheit zwischen Musik und Bühnenhandlung gewährleisten zu können, nahm Mahler bei der Realisierung des Fidelio wie auch in zahlreichen anderen Produktionen die Doppelfunktion des regieführenden Dirigenten ein und setzte zudem im Hinblick auf die visuelle Ausgestaltung der Bühne auf eine neue Art der Zusammenarbeit: Einen idealen Partner bei der Umsetzung wechselseitiger Durchdringung von Musik und Szene fand Mahler im theaterfremden Maler und Grafiker Alfred Roller, einem Gründungsmitglied der >Sezession` und Mitherausgeber der bedeutenden Kunstzeitschrift Ver Sacrum. Die Kollaboration fußte auf der grundlegenden Absicht, eine »einvernehmliche Konzeption« und »aufeinander bezogene Ausführung von Regie, Inszenierung und Bühnengestaltung« zu erreichen und so die in den Konventionen des I9. Jahrhunderts erstarrte Operntradition zu erneuern. Stimmungen, die durch die Musik erweckt wurden, sollten auf der Bühne auch visuell zum Ausdruck gebracht werden. So ersetzte Roller die traditionell streng realistischen Bühnendekorationen durch stilisierte szenische Räume, in denen erstmals Licht und Farbe und somit auch farbiges Licht als expressive Mittel eingesetzt wurden (Abbildung I).9

5 Vgl. Paul Robinson: Ludwig van Beethoven. »Fidelio«, Cambridge 1996 (Cambridge Opera Handbooks), insb. S. 152,155 .

6 Vgl. Constantin Floros: Mahler als Visionär auf der Opernbühne, in: Musiktheater in Wien um Igoo. Gustav Mahler und seine Zeitgenossen, hg. von Carmen Ottner und Erich Wolfang Partsch, Tutzing 20r4, S. I4I-I49, hier S. I4I.

7 Wolfgang Greisenegger: Neukonzeption des Szenenraumes um 1900. Anton Brioschi, Heinrich Lefler, Alfred Roller, in: Musiktheater in Wien um I900, S. I5I-160, hier S. I53.

8 Franz Willnauer: Gustav Mahlers »Regietheater«. Zur Rezeptions- und Wirkungsgeschichte der Mahlerschen Opernreform, in: Musiktheater in Wien um I900, S. I6I-I75, hier S. I71, vgl. auch S. I63-I66.

9 Vgl. Evan Baker: A Break in the Scenic Traditions of the Vienna Court Opera. Alfred Roller and the Vienna Secession, in: The Great Tradition and Its Legacp. The Evolution of Dramatic and Musical Theater 
Welch symbolhafte Bühnenstimmungen Rollers gezielter Umgang mit den Elementen Raum, Licht und Farbe in Einklang mit Mahlers Regieführung hervorrufen konnte, lässt sich für die Neuinszenierung des Fidelio von I904 etwa in den Beschreibungen des Zeitgenossen Oscar Bie nachvollziehen, der als Opernkritiker und Privatdozent für Kunstgeschichte ı9ıо über die Mahler-Roller'schen Arbeiten in Wien berichtete. Den Chor der Gefangenen (»O welche Lust«) im ersten Akt, der in gängigen Inszenierungen in voller Chorstärke und für gewöhnlich im Halbkreis formiert als Bravourstück zum Besten gegeben wurde, beschrieb Bie mit folgenden Worten:

»Die zweite Hälfte [des ersten Aktes] geht [...] im Gefängnishof vor sich, der in dunklem Licht und finsteren Massen gehalten ist, nur links oben ist ein Ausschnitt, durch den blauer Himmel und frisches Grün hereinlugt. Die Architektur ist meisterhaft, die symbolische Stimmung zwingend. Die Gefangenen kommen aus einem tiefen Loch unten im Hintergründe, und gehn nach links durch: wie wunderbar wirkt dieser Chor [...], wenn er sich sachte entwickelt und beim Anruf der Freiheit mit hochgestreckten Händen sein Crescendo singt, um unter den Augen eines Inspizienten, der von einer oberen Galerie in den Hof herabsieht, wieder ängstlich zu verstummen. «

Mahler hatte den Chor in seiner Größe stark reduziert, ließ die Sänger nacheinander aus der Erde empor kriechen und so die verzweifelte Lage der Unterdrückten sichtbar werden. ${ }^{\text {II }}$ Die Stimmung der zu Beginn des zweiten Akts folgenden Kerkerszene wirkt vor dem Hintergrund, dass die Bühne bis dahin traditionellerweise so gut ausgeleuchtet war, dass die Singenden für die Zuschauer stets deutlich zu sehen waren, besonders eindrücklich.

»Das Gefängnis Florestans treibt uns [...] weiter ins Dunkel hinein. Es ist undurchdringlich finster. Rocco und Fidelio sollten mit der Laterne in die Zisterne hinabsteigen, damit dämonische Schlagschatten sich an der Felsdecke bewegen. Die Schlußszene endlich bringt uns wieder ins Licht - dies Beethovensche ,vom Dunkel ins Licht $<$ ist stets sehr suggestiv betont ${ }^{\mathrm{I}}{ }^{\mathrm{I}}$

Dass die strahlende Darstellung der Schlussszene im Kontrast zur dunkel gehaltenen Kerkerszene befreiende Wirkung erzielte, liegt auf der Hand.

Wenngleich die Neuerungen der Mahler'schen Opernreform anfänglich sehr gemischt aufgenommen wurden und auf Widerstand aus den eigenen Reihen sowie von-

in Austria and Central Europe, hg. von Michael Cherlin, Halina Filipowicz und Richard L. Rudolph, New York/Oxford 2003 (Austrian history, culture, and society, Bd. 4), S. 237-245. Oscar Bie, zit. nach Karl-Josef Müller: Mahler. Leben, Werke, Dokumente, Mainz 2010 ('I988), S. 537 .

11 Vgl. La Grange: Gustav Mahler, Bd.3, S. 4. La Grange zufolge berichteten Zeitzeugen gar von bloß acht Sängern, was unter praktischen Gesichtspunkten hinsichtlich der dynamischen Balance in Bezug auf das Orchester eher unrealistisch scheint. Allerdings waren es bestimmt weniger Sänger als in Felix Weingartners Produktion von I908, von der Alfred Roller berichtet, Weingartner habe den Gefangenen-Chor auf vierzig Sänger ergänzt. Alma Mahler: Gustav Mahler. Erinnerungen und Briefe, Amsterdam I949 (' ${ }^{\mathrm{I}}$ 940), S. 422.

12 Müller: Mahler, S. 537 . 
seiten der Kritik und der Wiener Gesellschaft stießen, war diese Mise en Scène des Fidelio zum Ende von Mahlers Amtszeit 1907 kaum mehr wegzudenken.

Als Felix Weingartner im Januar I908 als Nachfolger Mahlers an der Hofoper seinen Einstand mit einer Aufführung des Fidelio in seiner eigenen Einrichtung gab, lösten die Abweichungen von der Mahler'schen Inszenierung heftige Kontroversen im Zuschauerraum aus. Die außerordentliche Missgunst, die Weingartner durch das Verwerfen der Mahler'schen Anlage des Fidelio zugunsten seiner eigenen entgegenschlug, wirkte sich auch auf die Meinung der Presse aus, sodass er für seine ganze Amtszeit - abgestempelt als »Feind Mahlers « ${ }^{13}$ - aus den Reihen der Anhänger seines Amtsvorgängers schlechte Kritiken erhielt. Bald entstand so in der öffentlichen Meinung ein Bild, das dem avantgardistischen Mahler einen eleganten, aber wenig innovativen Weingartner entgegensetzte und das sich mitunter bis heute erhalten hat. ${ }^{\text {I4 }}$

Wie für Mahler, so hatte auch für Weingartner der Einklang von Musik und szenischer Handlung auf der Theaterbühne oberste Priorität. Hiervon zeugen detaillierte Regieanweisungen, szenografische Skizzen und Anmerkungen in seinem Notenmaterial. ${ }^{5}$ Weingartner war der Ansicht, die »szenischen Vorgänge« seien in Fidelio vom Komponisten »ebenso klar vorgezeichnet wie in Wagners Werken«. Jegliche Experimente vonseiten des Regisseurs seien daher "geradezu ein Frevel ${ }^{\mathrm{I}}{ }^{6}$ Er schätzte Rollers Arbeit und beschäftigte ihn auch weiterhin als Ausstattungschef der Hofoper. ${ }^{17}$ Seine Anpassungswünsche am Bühnenbild zu Fidelio beabsichtigten in erster Linie die Wiederherstellung des Beethoven'schen Originals in der Endfassung von I8I4, an dem sich auch das Bühnenbild orientieren sollte. Roller berichtete in einem Brief vom 22. Januar I908 an Gustav Mahler von den Modifikationen am Bühnenbild (Abbildung 2):

»Akt r: Gefängnishof. Vorne rechts Roccos Wohnung. Davor ein gemütliches Plätzchen mit Blumen. Rechts dann weiter die zwei Türen zu den Gefängnissen. Hintergrund, Mitte das grosse Eingangstor mit einem Oberlichtgitter, durch das die blaue Luft hereinsieht. Hinter dem Tor die Bastion vom früheren letzten Bild. Links neben dem Tor in einem Torturm die Stube Jaquinos. Weiter links nach

13 Felix Weingartner: Lebenserinnerungen, Bd. 2, Zürich/Leipzig I929, S. I60.

14 Vgl. zum Verhältnis der beiden Dirigenten Gustav Mahler und Felix Weingartner auch Matthias Schmidt: Die »Selbstschöpfung « der Moderne. Felix Weingartner und Gustav Mahler als Dirigenten, in: Im Mass der Moderne, S. 35I-386.

15 Vgl. die zwei Klavierauszüge (Peters-Ausgabe) im Nachlass Felix Weingartners in der Universitätsbibliothek Basel. Einer der beiden Klavierauszüge wurde von Carmen Weingartner-Studer mit Eintragungen nach den Angaben ihres Mannes versehen, die sich auf eine Produktion des Fidelio am 8. Mai I940 beziehen. Der andere, etwas spärlicher bezeichnete Klavierauszug enthält Anmerkungen von Felix Weingartner selbst.

16 Felix Weingartner: Lebenserinnerungen, Bd. 3, o. S., unveröffentlichtes Typoskript, Nachlass Felix Weingartner, Universitätsbibliothek Basel.

17 Vgl. Müller: Mahler, S. 539. 


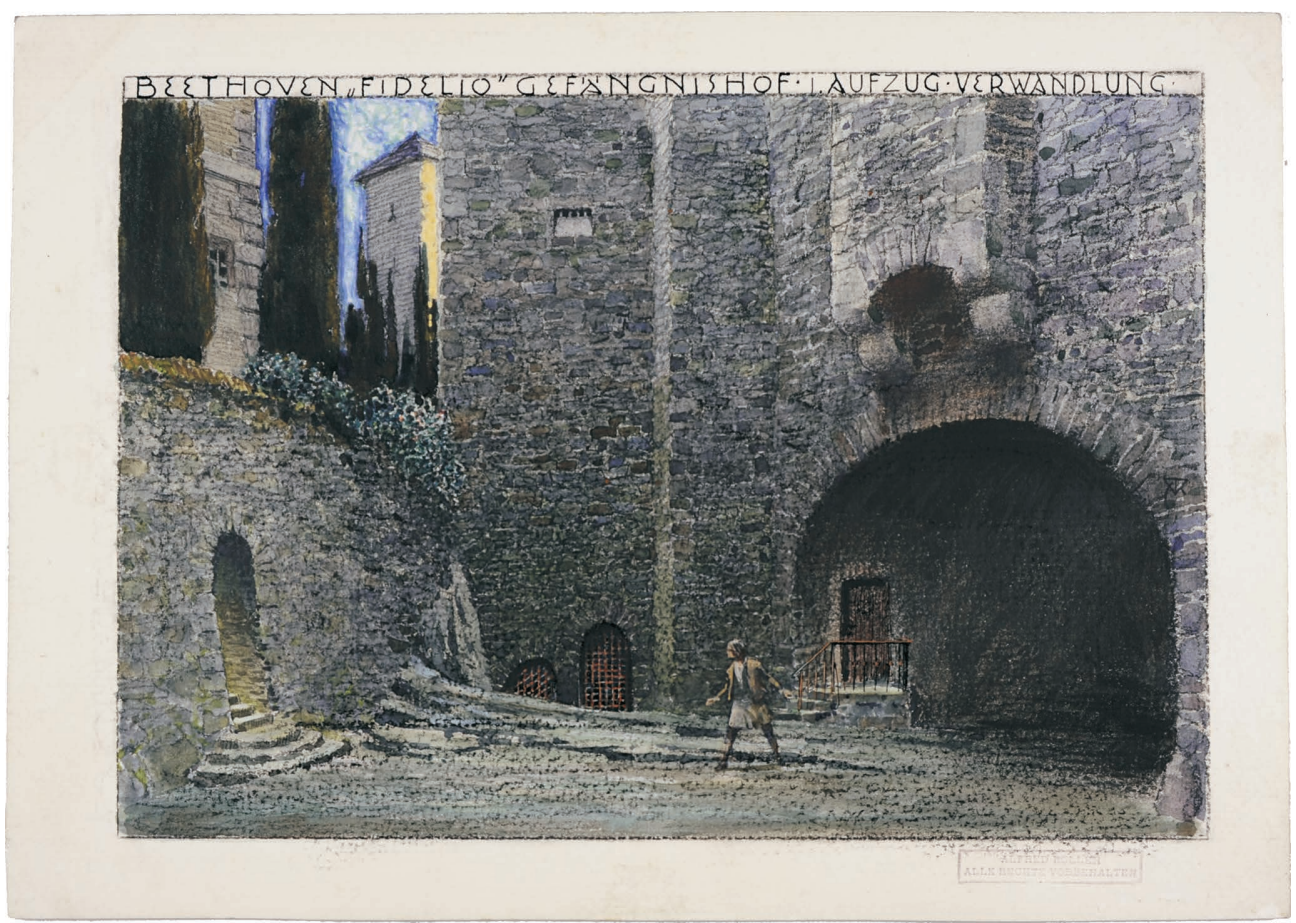

A B B ILDUNG I Bühnenbildentwurf der zweiten Szene des ersten Akts für die Fidelio-Produktion 1904 mit Gustav Mahler. Zeichnung von Alfred Roller im Nachlass Alfred Roller, кнм-Museumsverband, Theatermuseum Wien

\section{ERSTER AUFZUG}

Der Hof des Staatsgefängnisses. Im Hintergrund das Haupttor und eine hohe Wallmauer, über welche Bäume hervorragen. Im geschlossenen Tor selbst ist eine kleine Pforte, die für einzelne Fußgänger ge offnet wird. Neben dem Tor das Stübchen des Pförtners. Die Kulissen, den Zuschauern links, stellen die sind mit Eisen beschlagen und mit starken Riegeln verwahrt. In der vordersten Kulisse ist die Tür zur Wohnung des Gefangenwärters. Rechts stehen Bäume mit eisernen Geländern eingefabt, welche nebst einem Gartentor den Eingang des Schloßgartens bezeichnen.

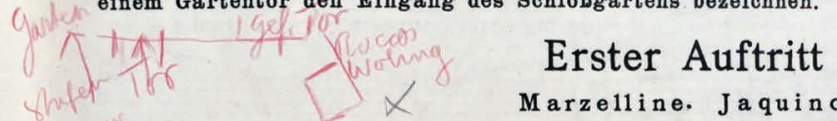

\section{Marzelline. Jaquino.} - robar, alliven

(M arzelline plättet vor ihrer Tür Wäsche, neben ihr steht ein Kohlenbecken, in dem sie den Stahl wärmt. Jaquino hält sich nahe bei seinem Stübchen, öffnet die Tür mehreren Personen, die ihm Pakete übergeben, welche er in sein Stübchen legt.)

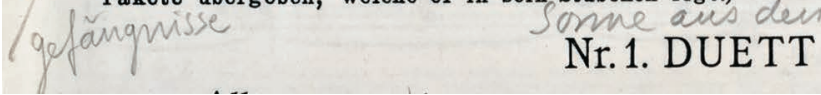

A в в ILDUNG 2 Skizze der Bühnenanordnung des ersten Akts. Klavierauszug von Kurt Soldan mit Anmerkungen von Carmen Weingartner-Studer nach Angaben von Felix Weingartner. Die Eintragungen beziehen sich auf eine Produktion von 1940, die - insbesondere in Bezug auf die Zeichnung mit rotem Farbstift - jedoch weitestgehend mit Weingartners erster Fidelio-Produktion 1908 an der Wiener Hofoper übereinzustimmen scheint. Die in der Skizze mit Bleistift markierten Gefängnisse hat Weingartner in seiner späteren Produktion dem Original folgend allerdings auf die linke Seite verlegt. U B Basel, NL 343 (Nachlass Carmen Weingartner-Studer), Schachtel Nr. 426, Nr. 19 (Inventarnummer), S. 15 
vorne der Eingang zum Garten und Schloss. Dieses letzte Stück blieb alt, alles andere in dem Hof wurde neu, da ich sonst nicht gewusst hätte, wie ich das freundliche Häuschen Roccos mit den pechschwarzen Mauern von früher im Licht hätte zusammenarbeiten sollen. So sind also auch die

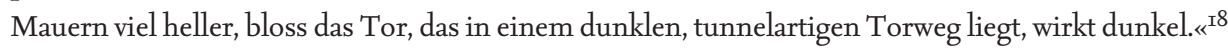

Der erste Akt sollte bei Weingartner wieder im Gefängnishof spielen und der Einschub der dritten Leonoren-Ouvertüre rückgängig gemacht werden. Der Wegfall der Ouvertüre als Intermezzo im zweiten Akt hatte zum allgemeinen Missfallen des Publikums aber die Konsequenz, dass für Rollers ursprüngliche - und grundsätzlich auch von Weingartner geschätzte - Schlussdekoration nun die benötigte Umbauzeit fehlte. Weingartner stellte jedoch die Unversehrtheit des Beethoven'schen Werks an oberste Stelle und verurteilte jeden Eingriff, der den Gang der Oper unterbrach, als »schwere[n] Fehler«, die Störung der Handlung nach der Kerkerszene jedoch geradezu als unentschuldbare »Versündigung« am Werk, ${ }^{\text {I9 }}$ weil hierdurch die Spannung vor Erreichen des »natürlichen Höhepunkt[es]« der Oper im Finale nachließ. ${ }^{20}$ Zusätzlich verstärkt wurde dieser Spannungsabfall nach Weingartners Ansicht dadurch, dass die Ouvertüre in derselben Tonart wie das Finale erklang. Um während der Oper die »gewaltige Steigerung von der ersten bis zur letzten Note ohne Störung zur Geltung« zu bringen, hielt er es gar für angebracht, den Fidelio ganz ohne Pause zu geben und so die Verwandlung der Szene jeweils zur fortlaufenden Musik bei geschlossenem Vorhang vonstatten gehen zu lassen. ${ }^{2 \mathrm{I}}$ Eine weitere Maßnahme zur Intensivierung des Spannungsverlaufs stellte die Reduktion gesprochener Dialoge auf handlungsrelevante Passagen dar - eine Praxis, die Weingartner auch bei anderen Singspielen wie etwa Mozarts Zauberföte anwandte (Abbildung 3). ${ }^{22}$

Als Opernintroduktion hielt Weingartner rein theoretisch jede der vier Ouvertüren für denkbar, wobei seine Präferenz auf der zweiten Leonoren-Ouvertüre lag, die Beethoven der Uraufführung der ersten Opernfassung zugedacht hatte. Sein Interesse an der zweiten Ouvertüre führte ihn zusammen mit wissenschaftlichen Mitarbeitern des Verlagshauses Breitkopf \& Härtel zu einem ausführlichen Quellenstudium an Beethovens Manuskripten, über welches er I927 in der Neuen Freien Presse detailliert Bericht erstattete.

Alfred Roller, zit. nach Mahler: Gustav Mahler, S. 4I5f. Im Unterschied zur Anweisung in Beethovens Partitur kommen die Gefängnisse bei Roller rechts und der Schlossgarten links zu liegen. Grund für die seitenverkehrte Anlage dürften praktische Überlegungen bei der Umarbeitung des MahlerRoller'schen Bühnenbilds gewesen sein.

19 Felix Weingartner: Die Ouvertüre zu »Fidelio«, in: ders.: Akkorde. Gesammelte Aufsätze, Leipzig I9I2, S. 8I-85, hier S. 82. Felix Weingartner: „Fidelio«-die Oper der Konzessionen, in: Neue Freie Presse vom 26. Mai 1935, S. II. Weingartner: Die Ouvertüre zu »Fidelio«, S. 82 f. Nachlass von Felix Weingartner, in: Notenlese. Musikalische Aufführungspraxis des I9. und frühen 20. Jahrhunderts in Basel, Basel 2013, S. I77-I89. 


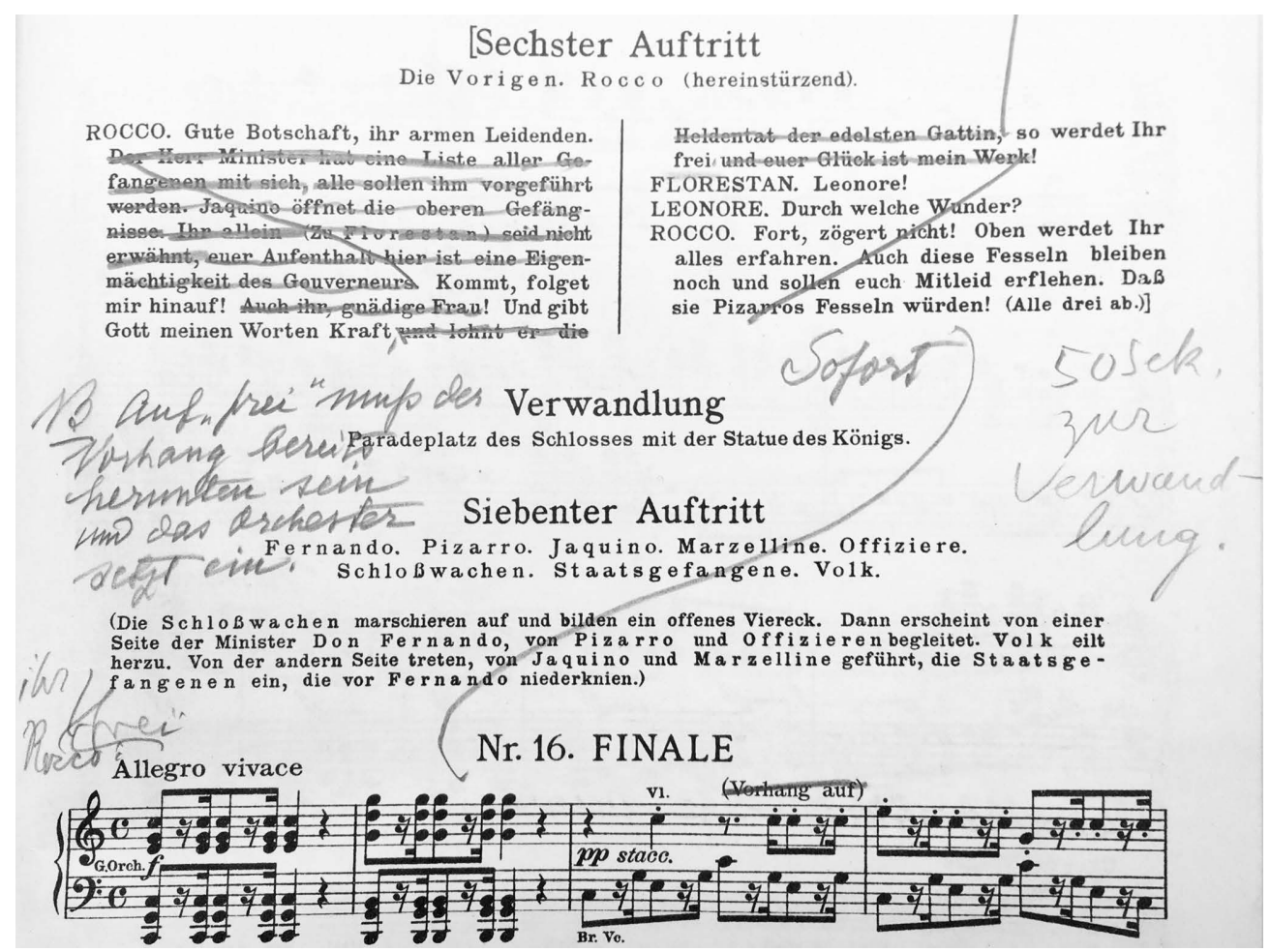

A B B ILDUNG 3 Textkürzungen zur Straffung der Handlung und Regieanweisungen betreffs der Verwandlung des Bühnenbilds von der Kerkerszene zum Finale auf dem Schlossplatz. Klavierauszug von Kurt Soldan mit Anmerkungen von Felix Weingartner. U B Basel, NL 343 (Nachlass Carmen Weingartner-Studer), Schachtel Nr. 425, Nr. 15 (Inventarnummer), S. 159

Neben innermusikalischen Gründen überzeugte ihn in diesem Kontext auch die Aussage des selbsternannten Beethoven-Vertrauten Anton Schindler, dass sich »Beethoven nur ungern von der zweiten Ouvertüre zugunsten der dritten « getrennt habe, die der Komponist selbst »mit einem echt Beethovenschen Scherzwort als >vermeistert< bezeichnet haben soll «. ${ }^{23}$ Gerade die zweite Leonoren-Ouvertüre bereitete als musikalische Eröffnung in den Augen Weingartners durch ihre Ausdrucksstärke, ihre »dramatische Kraft und Deutlichkeit « ${ }^{24}$ vorzüglich auf die folgende Handlung der Oper vor, während die dritte Ouvertüre »weit über das Theatralisch-Dramatische in das Gebiet des Episch-Symphonischen hinein « wachse und dadurch das Spannungsmoment der Handlung ersterbe. ${ }^{25}$

Tatsächlich aufgeführt hat Weingartner den Fidelio in seiner Wunschanordnung sicherlich während der ersten Periode seiner Zeit als Direktor der Wiener Hofoper I9o8I9II. Als er I935/36 dieses Amt abermals bekleidete, »nötigten [ihm die Umstände] eine

23 Felix Weingartner: Die zweite »Leonoren«-Ouvertüre, in: Neue Freie Presse vom 27. März I927, S. 33 f., hier S. 34 .

24 Weingartner: Die Ouvertüre zu »Fidelio«, S. 83.

25 Weingartner: Die zweite »Leonoren«-Ouvertüre, S. 34. 
Konzession ab«, sodass er bei seinem Antritt die wiederum existente szenische Trennung des ersten Akts in zwei Teile akzeptierte und auch die Fidelio-Ouvertüre als Einleitung stehenließ. Ebenso wollte er das Publikum »nicht um den Genuß« der großen LeonorenOuvertüre bringen. Sie wurde jedoch, durch eine Pause von der Oper losgelöst, zu Beginn des Abends gegeben, ${ }^{26}$ wie es vor Mahlers Ära bereits Usus gewesen war. Diese Aufführungsvariante hinterließ in der Presse einhellig den Eindruck eines Kompromisses, »von dem kaum anzunehmen ist, daß [er] die Verfechter der Mahlerschen Inszenierungsidee befriedigen « konnte. ${ }^{27}$

Dass die Einheit von Text, Musik und Bühnengeschehen nicht nur für Mahler, sondern auch für Weingartner oberstes Gebot war, ist der Verankerung beider Interpreten im Geist der Jahrzehnte um I9o0 zuzuschreiben. Indem Weingartner seinen Fidelio ohne unbedachte Übernahme der bestehenden Anlage seines Vorgängers in der eigenen Lesart umsetzte, zog er mit Mahler hinsichtlich der Eigenständigkeit seines Ansatzes gleich. Wie Mahler kämpfte auch Weingartner stets gegen leichtfertiges Weiterführen von Traditionen und stellte die individuelle Lektüre des Notentextes über die Rücksicht auf bestehende Erwartungen. ${ }^{28}$ Hätte Weingartner den Fidelio genau so übernommen, wie er ihm von Mahler hinterlassen wurde, so hätte er sich zu Recht den Vorwurf gefallen lassen müssen, der im Schlagwort »Tradition ist Schlamperei« mitschwingt. ${ }^{29}$ In anderen Aspekten unterscheiden sich Mahler und Weingartner in ihrem Verständnis eines werkgetreuen Interpretierens jedoch wesentlich.

Für Mahler hatte Werktreue nichts mit historischer Treue zu tun. Vielmehr ging es ihm um Wirkungstreue, die ihn dazu veranlasste, die Oper zur Entfaltung der ihr wahrhaft innewohnenden Größe einer Umgestaltung unter den ästhetischen Maximen seiner Zeit zu unterziehen. Für Weingartner hingegen war Werktreue nicht von stilistischer Treue zu trennen. Eine »stilvolle Wiedergabe «bedeutete für ihn, nicht zu aktualisieren, sondern sich in die Komposition zu versenken und eine »Sensibilität für die historische Position des zu interpretierenden Werkes zu entwickeln «.3०

Felix Weingartner: Wie ich den »Fidelio« sehe, in: Der Morgen vom I5. April I935, S. Ir.

27 [Anonym]: Weingartner und der »Fidelio«, in: Neues Wiener Journal vom i5. Oktober I935, S. ro; vgl. auch Ludwig Karpath:»Fidelio« unter Weingartner. (Zur morgigen Aufführung im Operntheater), in: Österreichische Zeitung am Abend vom I8. Mai 1935, Zeitungsausriss o. S., Nachlass Felix Weingartner, Universitätsbibliothek Basel. In letzterem Artikel steht fälschlicherweise, dass Weingartner nur die Fidelio-Ouvertüre (»kleine E-Dur Ouvertüre«) als der Oper angemessen betrachtete und die »sogenannten drei Leonoren-Ouvertüren [...], um Beethovens Willen zu befolgen, überhaupt nicht«zuließ. Vgl. Reinhard Kapp: »Tradition« und »Schlamperei«. Mahlers Einsatz: Bedingungen und Konsequenzen, in: Die Wiener Jahrhundertwende. Einflüsse, Umwelt, Wirkungen, hg. von Jürgen Nautz und Richard Vahrenkamp, Wien I993 (Studien zu Politik und Verwaltung, Bd. 46), S. 650-673. 
Der Umstand, dass Weingartner das Einfügen einer zweiten Ouvertüre grundsätzlich ablehnte, um die von Beethoven intendierte Endfassung zu erhalten, zeugt ebenso von seinem Bestreben nach Texttreue wie seine eingehende Beschäftigung mit den Quellen zur zweiten Leonoren-Ouvertüre. Seine Absicht, dem Beethoven'schen Notentext weitgehend zu folgen, ist verglichen mit seinen dirigierenden Zeitgenossen außergewöhnlich: Mahlers Erweiterung der Oper um eine zweite Ouvertüre als Entracte, die damit einhergehende Zweckentfremdung eines zur Einleitung komponierten Musikstückes als Zwischenakt- oder Umbaumusik und die szenische Zweiteilung des ersten Akts stießen hinsichtlich Texttreue bei kaum einem anderen Dirigenten der damaligen Zeit auf Bedenken. Im Gegenteil - nicht nur Richard Strauss und Wilhelm Furtwängler übernahmen den Einschub der dritten Leonoren-Ouvertüre nach Mahlers Vorbild, sondern diese Praxis etablierte sich sogar zu einem Standard, an dem erst Ende des 20. Jahrhunderts im Zuge bühnentechnischer Fortschritte, die einen schnellen Wechsel auch komplexer Szenenbilder ermöglichten, ${ }^{3 \mathrm{I}}$ und einhergehend mit Bestrebungen der historisch informierten Aufführungspraxis gerüttelt wurde. Vor diesem Hintergrund scheint Weingartner mit seinem Verständnis von Werktreue seiner Zeit gleichsam voraus gewesen zu sein.

Die Neuerungen, die Mahler in seiner Kooperation mit Roller an der Wiener Hofoper herbeigeführt hatte, brachten nicht nur für die Aufführungsgeschichte des Fidelio im Speziellen offensichtliche Konsequenzen mit sich, sondern auch für die Entwicklung des Musiktheaters im Allgemeinen: So haben Mahlers Ansprüche an eine Inszenierung das Aufgabenfeld der Regie von einem Arrangieren von Auf- und Abtritten und szenischen Tableaus hin zu einem Ausdeuten und Erarbeiten des dramatischen Sinns mit den beteiligten Künstlerinnen und Künstlern erweitert und zu einer Professionalisierung des Regieberufs geführt. ${ }^{32}$ Ebenso wegweisend war Rollers Umgang mit den Gestaltungselementen Licht, Farbe und Raum, die heute als selbstverständliche Mittel von Ausstattung und Szenografie gelten. Beethovens Fidelio ist durch Mahlers Akzentverschiebung vom Singspiel, in welchem die liebende Ehefrau in Idealgestalt aufopfernd den Gatten rettet, 33 zum epischen Drama mit politischer Aussage geworden, in dem Menschlichkeit und Freiheit über tyrannische Gewalt siegen. Auch dieser Aspekt aus Mahlers Interpretation hat sich im Wesentlichen bis heute gehalten und bleibt für die aktuelle Beethoven-Interpretation von Bedeutung.

Moosmüller: Stiltreues und wirkungstreues Interpretieren, S. 340 f. Moosmüller erwähnt etwa auch den Umgang Mahlers und Weingartners mit Retuschen in Beethovens 9. Sinfonie. Vgl. Robinson: Ludwig van Beethoven, S. I8If.

Vgl. Robinson: Ludwig van Beethoven, S. I50. 


\section{Inhalt}

Vorwort 8

INTERPRETATION - BEGRIFF, METHODE, PRAXIS

Laure Spaltenstein Interpretation als treue Übersetzung. Zur Frühgeschichte eines vieldeutigen Begriffs I5

Kai Köpp Von der Quelle zur Methode. Zum Entwurf einer historischen Interpretationsforschung $\quad 28$

Manuel Bärtsch >Interpretation‘. Beethovens Sonate A-Dur op. IOI in der Sicht von Eugen d'Albert und Frederic Lamond

Sebastian Bausch Klavierrollen als Interpretationsdokumente. Ein Erfahrungsbericht als Leitfaden für Einsteiger $\quad 7 \mathrm{I}$

Camilla Köhnken Beethoven-Auslegung zwischen Liszts »Deklamationsstil« und Bülows »Vivisektionsversuchen«. Auf den Spuren Liszt'scher Interpretationsideale in Hans von Bülows instruktiver Edition der Klaviersonaten Beethovens $\quad 92$

Neal Peres Da Costa Carl Reinecke's Performance of his Arrangement of the Second Movement from Mozart's Piano Concerto K. 488. Some Thoughts on Style and the Hidden Messages in Musical Notation

II4

Carolina Estrada Bascuñana Enrique Granados's Performance Style.

Visualising the Audible Evidence I5O

Lukas Näf Tempogestaltung in Weberns Sinfonie op. 2I I80

INTERPRETATION - AUFFÜHRUNGSGESCHICHTE

Christoph Moor "Ein so erklärtes Lieblingsstück der hiesigen Kunstfreunde«. Die Rezeptionsgeschichte der Jupiter-Sinfonie in Beethovens Wien

Luisa Klaus Objektive Bruckner-Interpretation? Zur Aufführung der Trio-Entwürfe für die Neunte Sinfonie I940 205

Chris Walton Von innen und von außen. Beethovens Neunte Sinfonie und die $>$ Wagner'sche< Dirigiertradition $\quad 2 \mathrm{I} 8$

Lena-Lisa Wüstendörfer Streit um Fidelio. Gustav Mahler und Felix Weingartner im Disput um Werktreue $\quad 238$

INTERMEZZO

Robert Levin Turning Point to Musical Modernity. Beethoven as Executor of the Legacy of C. P. E. Bach. Concert Lecture 249

INTERPRETATION - INSTRUMENTE, ANALYSE, EDITION

Martin Skamletz «Man hat diese Erweiterung des Tonumfanges seit ein paar Jahren an den Tasteninstrumenten sehr weit getrieben.« Der Umgang mit Grenzen beim späten Mozart und beim frühen Beethoven $\quad 263$ 
Stephan Zirwes Analyse und Interpretation. Adolph Bernhard Marx' Beethoven-Analysen 29I

Michael Ladenburger Was können wir aus Originalhandschriften von Beethoven für eine angemessene Interpretation lernen? 30I

Federica Rovelli Die Skizzenbuch-Ausgaben und ihre mögliche digitale Zukunft 3 I7

Johannes Gebauer Interpretationspraktische Stemmatik. Philologische Methoden in der Interpretationsforschung am Beispiel annotierter Notenausgaben von Rodes 24 Capricen und Beethovens Violinkonzert

John Rink Chopin Copying Chopin 349

Tomasz Herbut Alexander Goldenweiser und Beethovens Sonate op. IIo- eine Spurensuche 366

INTERPRETATION - KREATIVE ANEIGNUNG

Thomas Gartmann Beethoven als sein eigener Interpret. Gedanken zur Bearbeitung der Klaviersonate op. I4/ז für Streichquartett

Ivo Haag Die Sinfonien von Johannes Brahms - (auch) Klaviermusik?

Michael Lehner Das Orchester auf dem Klavier. Welte-Klavierrollen von Gustav Mahler und Richard Strauss als interpretationsanalytische Quellen

Roger Allen “That Is What Music Really Is". Richard Wagner's Reception of Beethoven's Piano Sonata in A Major Op. IOI $43 \mathrm{I}$

Daniel Allenbach Eine heroische< Neunte? Dmitri Schostakowitschs Neunte Sinfonie im Vergleich mit Ludwig van Beethoenvs Sinfonien Nr.3 und 9 44I

Simeon Thompson Beethoven und der Zweite Weltkrieg in der künstlerischen Reflexion der Nachkriegszeit. Stanley Kubricks A Clockwork Orange und Rolf Liebermanns Leonore 40/45 456

Michelle Ziegler Rettungsversuch im Jubiläumsjahr. Mauricio Kagels Aufarbeitung der Beethoven-Rezeption in der Ludwig van-Werkgruppe (I970) 465

Leo Dick Über den späten Beethoven zur >Postidentität،. Die Suche nach liminalen Räumen im gegenwärtigen Musiktheater am Beispiel von Matthias Rebstocks Berliner Produktion Büro für postidentisches Leben $\quad 476$

Elizabeth Waterhouse Choreographic Re-mix. William Forsythe's Trio (I996) and Beethoven's String Quartet No. I5 in a Minor Op. I32 487

László Stachó "Gradus ad Parnassum".

The Purgatory of Instrumental Technique

Namen-, Werk- und Ortsregister 522

Die Autorinnen und Autoren der Beiträge 


\section{Rund um BeEthoven \\ Interpretationsforschung heute • \\ Herausgegeben von Thomas}

Gartmann und Daniel Allenbach 


\section{MUSIKFORSCHUNG DER Hochschule der KÜnste Bern Herausgegeben von Martin Skamletz und Thomas Gartmann Band 14}


0 Dieses Buch ist in gedruckter Form im Dezember 2019 in erster Auflage in der Edition Argus in Schliengen/Markgräflerland erschienen. Gestaltet und gesetzt wurde es im Verlag aus der Seria und der SeriaSans, die von Martin Majoor im Jahre 2000 gezeichnet wurden. Gedruckt wurde es auf Eos, einem holzfreien, säurefreien, chlorfreien und alterungsbeständigen Werkdruckpapier der Papierfabrik Salzer im niederösterreichischen Sankt Pölten. Das Vorsatzpapier Caribic cherry wurde von Igepa in Hamburg geliefert. Rives Tradition, ein Recyclingpapier mit leichter Filznarbung, das für den Bezug des Umschlags verwendet wurde, stellt die Papierfabrik Arjo Wiggins in Issy-les-Moulineaux bei Paris her. Das Kapitalband mit rot-schwarzer Raupe lieferte die Firma Dr. Günther Kast aus Sonthofen im Oberallgäu, die auf technische Gewebe und Spezialfasererzeugnisse spezialisiert ist. Gedruckt und gebunden wurde das Buch von der Firma Bookstation im bayerischen Anzing. Im Internet finden Sie Informationen über das gesamte Verlagsprogramm unter www.editionargus.de, zum Institut Interpretation der Hochschule der Künste Bern unter www.hkb.bfh.ch/interpretation und www.hkb-interpretation.ch. Die Deutsche Nationalbibliothek verzeichnet diese Publikation in der Deutschen Nationalbibliografie; detaillierte bibliografische Daten sind im Internet über www.dnb.de abrufbar. (c) der zeitgleich erschienenen digitalen Version: die Autorinnen und Autoren, 20I9. Dieses Werk ist lizenziert unter einer Creative Commons Namensnennung-Nicht kommerziell 4.0 International Lizenz (CC BY-NC 4.o). DoI: https://doi.org/I0.26045/kp64-6I78 ISBN 978-3-93I264-94-9 